\title{
Magnetic fields and ionized gas in nearby late type galaxies ${ }^{\star}$
}

\author{
K. T. Chyży ${ }^{1}$, D. J. Bomans ${ }^{2}$, M. Krause ${ }^{3}$, R. Beck ${ }^{3}$, M. Soida ${ }^{1}$, and M. Urbanik ${ }^{1}$ \\ 1 Astronomical Observatory, Jagiellonian University, ul. Orla 171, 30-244 Kraków, Poland \\ e-mail: chris@oa.uj.edu.pl \\ 2 Astronomisches Institut, Ruhr-Universität-Bochum, 44780 Bochum, Germany \\ 3 Max-Planck-Institut für Radioastronomie, Auf dem Hügel 69, 53121 Bonn, Germany
}

Received 29 June 2006 / Accepted 3 November 2006

\begin{abstract}
Aims. In order to analyze the importance of the star formation rate in generating and amplifying magnetic fields in the interstellar medium we perform a deep continuum polarization study of three angularly large, late type spiral galaxies.

Methods. We obtained deep total power and polarization maps at $4.85 \mathrm{GHz}$ of NGC 4236, NGC 4656 and IC 2574 using the $100-\mathrm{m}$ Effelsberg radio telescope. This was accompanied by imaging in the $\mathrm{H} \alpha$ line. We also observed these objects at $1.4 \mathrm{GHz}$ to obtain their integrated fluxes at this frequency and to determine their radio spectra.

Results. All galaxies were found to possess weak but detectable total power emission at $4.85 \mathrm{GHz}$, coincident with regions of recent star formation as traced by bright $\mathrm{H} \alpha$ regions. The surface brightness of the radio-strongest object of our sample (NGC 4656) is comparable to the radio-weakest objects in a sample of more than 50 normally star-forming spiral galaxies for which measurements at $4.8 \mathrm{GHz}$ with the Effelsberg radio telescope are available. The surface brightness of the two other objects is even three times smaller. The fractional polarization of the 2 galaxies of our sample is less than $2 \%$, significantly lower than for spiral galaxies of intermediate types, suggesting that the magnetic fields are not only weaker, but also less ordered than in spiral galaxies. The radio spectra of galaxies in our small sample are indicative of a substantial fraction of thermal emission, with a higher thermal fraction than in spirals with high star formation rates (SFR), while the nonthermal emission in our sample is relatively weak compared to spiral galaxies. We propose an equipartition model where the nonthermal emission increases $\propto S F R^{\approx 1.4}$ and the ratio of nonthermal to thermal emission increases $\propto S F R^{\approx 0.4}$. The objects of our sample still follow the radio-FIR correlation of surface brightness of the total emission, extending it towards the lowest values measured so far.
\end{abstract}

Key words. galaxies: individual: NGC 4236 - galaxies: individual: NGC 4656 - galaxies: individual: IC 2574 galaxies: magnetic fields - radio continuum: galaxies - ISM: magnetic fields

\section{Introduction}

The generation of galactic magnetic fields requires strong dynamo action (Beck et al. 1996) driven by Coriolis forces caused by rapid disk rotation. As suggested by Chyży et al. (2000) rotation alone is not the only agent in generating the large-scale regular and random magnetic fields. In the classical dynamo theory as well as in more recent dynamo concepts (e.g. Blackman 1998; Schekochihin et al. 2004) the energy input from interstellar turbulence is required. Turbulence itself is believed to be powered by star-forming processes. A promising mechanism to generate quickly strong magnetic fields, even with weak Coriolis forces, is the dynamo mechanism based on the Parker instability (Kowal et al. 2003; Hanasz et al. 2004; Moss et al. 1999). This process depends directly on the formation of massive stars, because the instabilities grow due to the pressure of cosmic rays (CR) produced by supernovae. This model explains very well the stronger magnetic fields in rapidly star-forming spirals. On the other hand, this may mean that in galaxies forming stars very slowly the instabilities cannot produce widespread magnetic fields.

The above relation seems to be supported by observations of the low surface brightness irregular galaxy NGC 6822 by

* Based on the observations with the 100-m telescope at Effelsberg operated by the Max-Planck-Institut für Radioastronomie (MPIfR) on behalf of the Max-Planck-Gesellschaft.
Chyży et al. (2003). This very slowly star-forming galaxy shows only weak signs of global magnetic fields. Until now no detailed radio continuum study of such objects was made. In particular it is not known whether at the low end of the surface brightness distribution the magnetic field strength decreases continuously in some proportion to the star formation rate or drops suddenly to zero below a certain threshold of star-forming activity.

In a search for such a threshold we undertook a study of three late type galaxies - NGC 4236, NGC 4656, IC 2574 - showing rotational and other properties intermediate between spirals and irregulars (Table 1). We have chosen galaxies of substantial inclination to ensure a long pathlength through the disk and hence a higher observed surface brightness. They all have rather low surface brightnesses with different star-forming properties. While NGC 4236 has a star-forming activity symmetrically distributed over the disk, NGC 4656 shows almost all star formation concentrated in one of the disk halves. The third galaxy, IC 2574, has a very low star formation level, restricted to one region in the disk outskirts. NGC 4236 and IC 2574 belong to the M 81 group and both show similar color-magnitude diagrams (Karachentsev et al. 2002). IC 2574 seems to be optically very diffuse and does not reveal any obvious nucleus (Ho et al. 1998). NGC 4656 belongs to the group of NGC 4631, to which the distance is only $47 \mathrm{kpc}$ and in which direction some H I filaments are observed (Rand 1994). 
Table 1. Basic properties of NGC 4236, NGC 4656 and IC 2574 (mainly from the LEDA database).

\begin{tabular}{|c|c|c|c|}
\hline & NGC 4236 & NGC 4656 & IC 2574 \\
\hline $\mathrm{RA}_{2000}$ & $12^{\mathrm{h}} 16^{\mathrm{m}} 43^{\mathrm{s}} .1$ & $12^{\mathrm{h}} 43^{\mathrm{m}} 58^{\mathrm{s}} .2$ & $10^{\mathrm{h}} 28^{\mathrm{m}} 21^{\mathrm{s}} .5$ \\
\hline $\operatorname{Dec}_{2000}$ & $+69^{\circ} 27^{\prime} 51^{\prime \prime}$ & $+32^{\circ} 10^{\prime} 14^{\prime \prime}$ & $+68^{\circ} 24^{\prime} 41^{\prime \prime}$ \\
\hline Inclination $^{a}$ & $75^{\circ b}$ & $83^{\circ} \mathrm{C}$ & $77^{\circ d}$ \\
\hline Position Angle & $162^{\circ}$ & $35^{\circ}$ & $50^{\circ}$ \\
\hline Morphol. Type & SBdm & $\mathrm{SBm}$ & $\mathrm{SABm}$ \\
\hline Optical diameter $D_{25}$ & 23.4 & 13.5 & $13 \cdot 5$ \\
\hline Distance $[\mathrm{Mpc}]$ & $4.45^{e}$ & $7.5^{c}$ & $4.02^{e}$ \\
\hline$B$-magnitude & $10^{\mathrm{m}} 06$ & $11^{\mathrm{m}} \cdot 35$ & $10^{\mathrm{m}} 80$ \\
\hline Absolute $B$-magnitude & $-18 \cdot 01$ & -20.57 & $-16^{\mathrm{m}} 95$ \\
\hline Mean surface brightness $\left[\mathrm{mag} / \operatorname{arcsec}^{2}\right]$ & $24 . \mathrm{m} 61$ & 24.27 & 24.52 \\
\hline $\mathrm{H}$ I mass $\left[M_{\odot}\right]^{c}$ & $1.5 \times 10^{9}$ & $5 \times 10^{9}$ & $6.7 \times 10^{8}$ \\
\hline Rotation velocity (from $\mathrm{HI}\left[\mathrm{km} \mathrm{s}^{-1}\right]$ ) & $\approx 85^{b}$ & $\approx 75^{c}$ & $67^{d}$ \\
\hline
\end{tabular}

${ }^{a} 0^{\circ}=$ face-on; ${ }^{b}$ Honma \& Sofue (1997); ${ }^{c}$ Rand (1994); ${ }^{d}$ Martimbeau et al. (1994); ${ }^{e}$ Karachentsev et al. (2002).

The galaxies are angularly large, with $\mathrm{D}_{25}$ ranging from $13^{\prime}$ to $23^{\prime}$ (see Table 1). Their expected radio surface brightness is too low to be detectable at centimeter wavelengths with the VLA even with the most compact configuration. On the other hand, they are large enough to be resolved at $4.85 \mathrm{GHz}$ with 2.5 HPBW resolution of the Effelsberg telescope which enables to identify large-scale star formation distributions. We decided to use this instrument and frequency for our study. To obtain detailed information on their star-forming properties, the galaxies were mapped in the $\mathrm{H} \alpha$ line with the 1-m telescope at Mt. Laguna Observatory. This kind of study also brings information about the ionized gas properties in slowly star-forming, late-type spirals and especially about the thermal free-free emission. To make another estimate of their thermal emission from the radio spectrum we measured also their integrated flux densities at $1.4 \mathrm{GHz}$ with the Effelsberg telescope. Confusion by unresolved background sources is severe at this frequency, but high resolution data are available at $1.49 \mathrm{GHz}$ (NVSS, FIRST, Condon 1987), enabling the subtraction of point sources unrelated to galaxies from our measurements.

\section{Observations and data reduction}

\subsection{Ho images}

The optical data for our galaxies were taken with the 1-m telescope at Mt. Laguna Observatory (operated by the San Diego State University, California) using a Loral/Lesser $2048^{2}$ pixel $\mathrm{CCD}$. The data reduction techniques were the same as described by Chyży et al. (2003) and performed in the standard way using the IRAF package. We applied small kernel Gaussian filters to both the $\mathrm{H} \alpha$ and the $R$ image to match the slightly different point spread functions. After this step the continuum subtraction was performed, as described in e.g. Bomans et al. (1997). The maps were calibrated to the absolute scale and corrected for the $\mathrm{N}$ II contribution.

\subsection{Radio continuum}

The total power and polarization observations were performed at $4.85 \mathrm{GHz}$ with 2!.5 HPBW resolution using the two-horn system in the secondary focus of the Effelsberg 100-m MPIfR telescope (Schmidt et al. 1993). The telescope pointing was corrected by making cross-scans of bright point sources at time intervals of about $1.5 \mathrm{~h}$. The flux density was calibrated on the highly polarized source $3 \mathrm{C} 286$. A total power flux density at $4.85 \mathrm{GHz}$ of $7.47 \mathrm{Jy}$ was adopted using the formulae by Baars at al. (1977). The polarized flux density was calibrated using the same factors as for total power, yielding a degree of polarization of $10.5 \%$ for $3 \mathrm{C} 286$, which is in good agreement with other published values (Tabara \& Inoue 1980). At $4.85 \mathrm{GHz}$ we observed NGC 4236, NGC 4656 and IC 2574 in the azimuth-elevation frame with field sizes of $40^{\prime} \times 30^{\prime}, 46^{\prime} \times 30^{\prime}$ and $46^{\prime} \times 36^{\prime}$, respectively. The pixel size and separation between scans of $1^{\prime}$ fulfill the necessary (Nyquist) sampling requirement. The scaning velocities were $30^{\prime} / \mathrm{min}$ for NGC 4236 and NGC 4656, and 50' $/ \mathrm{min}$ for IC 2574. We used the NOD2 data reduction package (Haslam 1974). By combining the information from the two horns, using the "software beam switching" technique (Morsi \& Reich 1986) followed by restoration of total intensities (Emerson et al. 1979), we obtained $I, Q$ and $U$ maps for each coverage of a given galaxy. Those channel maps with an excess signal along the scanning direction were removed from further analysis, resulting in 47 total power and 36 polarized channel maps for NGC 4236, 19 and 19 maps for NGC 4656, and 20 and 19 maps for IC 2574, respectively. All good maps were then combined using the spatial-frequency weighting method (Emerson \& Gräve 1988), followed by a digital filtering process, that removed the spatial frequencies corresponding to noisy structures smaller than the telescope beam. Finally the $I, Q$ and $U$ images were combined into the maps of total power, polarized intensity, polarization degree and polarization position angles. The rms noise levels in the final maps of total intensity are $0.4 \mathrm{mJy} / \mathrm{b} . \mathrm{a} ., 0.7 \mathrm{mJy} / \mathrm{b} . \mathrm{a}$. and $0.4 \mathrm{mJy} / \mathrm{b}$.a. for NGC 4236, NGC 4656 and IC 2574, respectively. The corresponding noise levels in polarized intensity are $0.04 \mathrm{mJy} / \mathrm{b} . a ., 0.07 \mathrm{mJy} / \mathrm{b}$.a. and $0.08 \mathrm{mJy} / \mathrm{b}$.a.

At $1.4 \mathrm{GHz}$ we used the single horn system in the primary focus of the Effelsberg 100-m telescope. IC 2574 was observed in a single band at $1.400 \mathrm{GHz}$ with a bandwidth of $20 \mathrm{MHz}$, whereas for NGC 4256 and NGC 4656 the band was splitted into two independent channels centered on $1.395 \mathrm{GHz}$ and $1.408 \mathrm{GHz}$, respectively, with a bandwidth of $14 \mathrm{MHz}$ each. We obtained 21 good quality coverages for NGC 4236 and 14 coverages for NGC 4656 with field sizes of $84^{\prime} \times 84^{\prime}$, respectively. For IC 2574 we got 12 coverages with a field size of $60^{\prime} \times 60^{\prime}$. The maps were scanned in alternating directions along RA and Dec. The pixel size and separation between the scans were set to $3^{\prime}$. The scanning velocity was $4^{\circ} / \mathrm{min}$ for NGC 4236 and NGC 4656 , and $2 \%$ min for IC 2574 . We obtained total power maps of the galaxies with an angular resolution of $9 ! 3 \mathrm{HPBW}$ and an rms noise of $5 \mathrm{mJy} / \mathrm{b} . \mathrm{a} ., 2 \mathrm{mJy} / \mathrm{b}$.a. and $8 \mathrm{mJy} / \mathrm{b}$.a. for $\mathrm{NGC} 4236$, NGC 4656 and IC 2574, respectively. 
Table 2. Confusion-corrected fluxes, spectral indices, observed and assumed nonthermal, as well as thermal fluxes and fractions of observed galaxies at $4.85 \mathrm{GHz}$.

\begin{tabular}{lrrcrrrrr}
\hline \hline Galaxy & $\begin{array}{r}S_{\text {tot }} \\
4.85\end{array}$ & $\begin{array}{r}S_{\text {tot }} \\
1.4\end{array}$ & $\alpha_{\text {obs }}$ & $\alpha_{\text {nt }}$ & $\begin{array}{r}S_{\text {th }}(4.85) \\
\text { radio } \\
{[\mathrm{mJy}]}\end{array}$ & $\begin{array}{r}f_{\text {th }}(4.85) \\
\text { radio }\end{array}$ & $\begin{array}{r}S_{\text {th }}(4.85) \\
\text { opt } \\
{[\mathrm{mJy}]}\end{array}$ & $\begin{array}{r}f_{\text {th }}(4.85) \\
\text { opt }\end{array}$ \\
\hline NGC 4236 & 23 & 48 & $0.59 \pm 0.15$ & 0.8 & 9.0 & 0.39 & 11.7 & $0.51 \pm 0.07$ \\
& & & & 0.9 & 11.6 & 0.50 & & \\
NGC 4656 & \multirow{2}{*}{42} & \multirow{2}{*}{92} & $0.63 \pm 0.12$ & 0.8 & 13.7 & 0.33 & 26.9 & $0.64 \pm 0.14$ \\
& & & & 0.9 & 18.9 & 0.45 & & \\
IC 2574 & & & & 1.0 & 22.9 & 0.55 & & \\
& & & & & & & \\
& & & $0.56 \pm 0.36$ & 0.8 & 4.2 & 0.45 & 6.5 & $0.68 \pm 0.06$ \\
& & & & 0.9 & 5.2 & 0.55 & & \\
\hline
\end{tabular}

Note: the uncertainties of optically derived $f_{\text {th }}$ result from various estimates of total extinction.

\section{Observational results}

Below we present the radio data separately for the individual galaxies. To find possible background sources contributing to the integrated flux densities we compared our total power maps at $4.85 \mathrm{GHz}$ with high-resolution ones at $1.49 \mathrm{GHz}$ from Condon (1987), NVSS and FIRST. In our $1.4 \mathrm{GHz}$ data the contribution from all point sources not obviously related to the galaxy was subtracted from our integrated flux densities. At $4.85 \mathrm{GHz}$ we applied the "best subtraction" (Chyży et al. 2003) at the positions of background sources to estimate their contribution at $4.85 \mathrm{GHz}$. These values were then used to derive the background-free fluxes at this frequency, listed in Table 2.

\subsection{NGC 4236}

NGC 4236 shows two groups of large H II regions at both ends of the disk (Fig. 1). They are embedded in diffuse $\mathrm{H} \alpha$ emission. The centre of the galaxy contains only small H II regions and much less diffuse emission. Some of the ionized gas clumps are found as far as $3^{\prime}$ or $3.7 \mathrm{kpc}$ (inclination corrected) from the disk plane. This is too far to belong to the disk. Hence, NGC 4236 clearly possesses extraplanar $\mathrm{H} \alpha$ emission from regions up in the halo.

The radio emission in NGC 4236 at $4.85 \mathrm{GHz}$ has a central peak in the region where no large groups of $\mathrm{H}$ II regions are found (Fig. 2). The NW cluster of $\mathrm{H}$ II regions corresponds to a radio extension while the $\mathrm{SE}$ one is associated with a separate source. The gap between this feature and the central peak corresponds to a region particularly deficient in $\mathrm{H} \alpha$ emission.

The polarized sources at $\mathrm{RA}_{2000}=12^{\mathrm{h}} 15^{\mathrm{m}} 30^{\mathrm{s}}, \mathrm{Dec}_{2000}=$ $69^{\circ} 24^{\prime}$ and at $\mathrm{RA}_{2000}=12^{\mathrm{h}} 15^{\mathrm{m}} 30^{\mathrm{s}}, \mathrm{Dec}_{2000}=69^{\circ} 34^{\prime}$ are composed of four point sources visible in Condon's (1987) and NVSS maps. They are certainly background objects. They do not interfere with the galaxy measurements at $4.85 \mathrm{GHz}$ but had to be removed from the $1.4 \mathrm{GHz}$ integrated flux. The polarized extension NE of the northernmost star-forming clump turned out to be a background source, as well. It was deleted in our integrated flux density determinations at both frequencies. The only detectable polarized signal not attributed to background sources comes from the central region of NGC 4236 (Fig. 3).

We note that Turner \& Ho (1994) found three point sources in the central region of NGC 4236 which may be radio supernovae. They may at least partly explain the polarization in the central region.

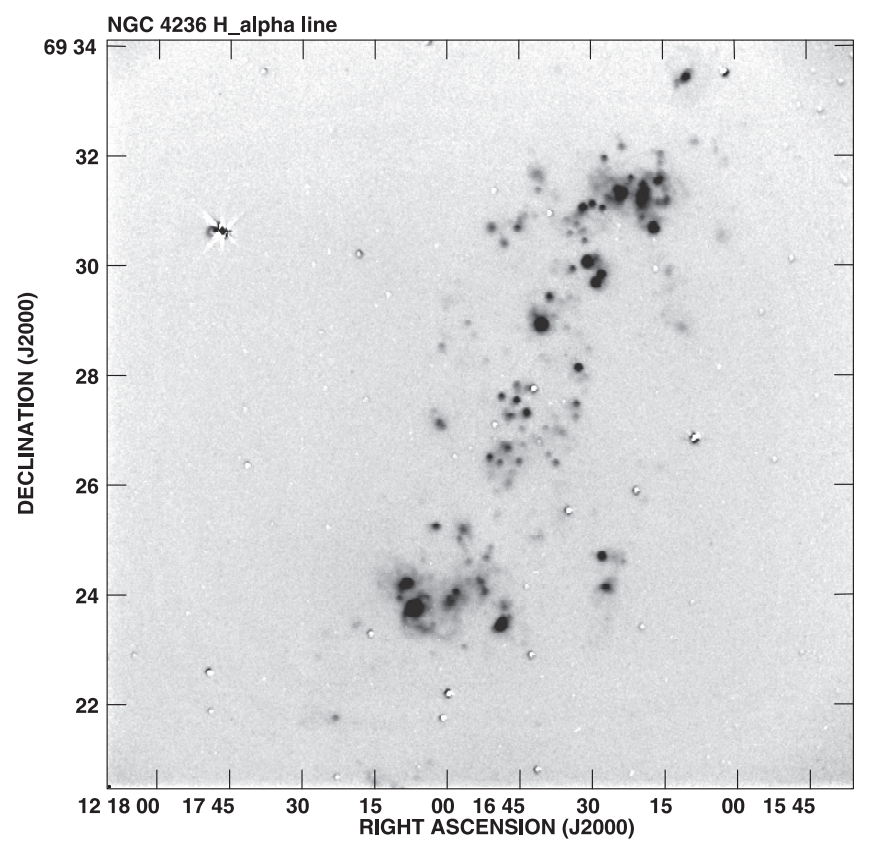

Fig. 1. The $\mathrm{H} \alpha$ image of NGC 4236 with the optical continuum subtracted.

Integration of the total power map in a polygonal region encompassing the detectable emission (without background sources) yields an integrated flux density of NGC 4236 at $4.85 \mathrm{GHz}$ of $23 \pm 3 \mathrm{mJy}$. Integration of the map of polarized intensity in the same area yields a polarized flux density of $0.37 \pm 0.13 \mathrm{mJy}$ which means 3 -sigma detection. The central peak gives $13 \mathrm{mJy} / \mathrm{b}$.a. in total intensity and $0.18 \mathrm{mJy}$ in polarized intensity which means detection at 4.5 sigma confidence level. Integration of the $1.4 \mathrm{GHz}$ map yields a total flux density (corrected for background sources) of $48 \pm 6 \mathrm{mJy}$.

\subsection{NGC 4656}

In NGC 4656 almost all $\mathrm{H} \alpha$ emission is concentrated in the NE part of the disk (Fig. 4). This region of the galaxy is populated by large groups of giant $\mathrm{H}$ II regions surrounded by diffuse emission. At the northeastern end of the disk they make a hook-like appendix, extending by more than $1^{\prime}$ to the east. The SW part of the disk shows very weak $\mathrm{H} \alpha$ emission and very few small $\mathrm{H}$ II regions. We also note a weak ridge of $\mathrm{H} \alpha$ emission running parallel to the disk plane at a distance of about $1^{\prime}$ 


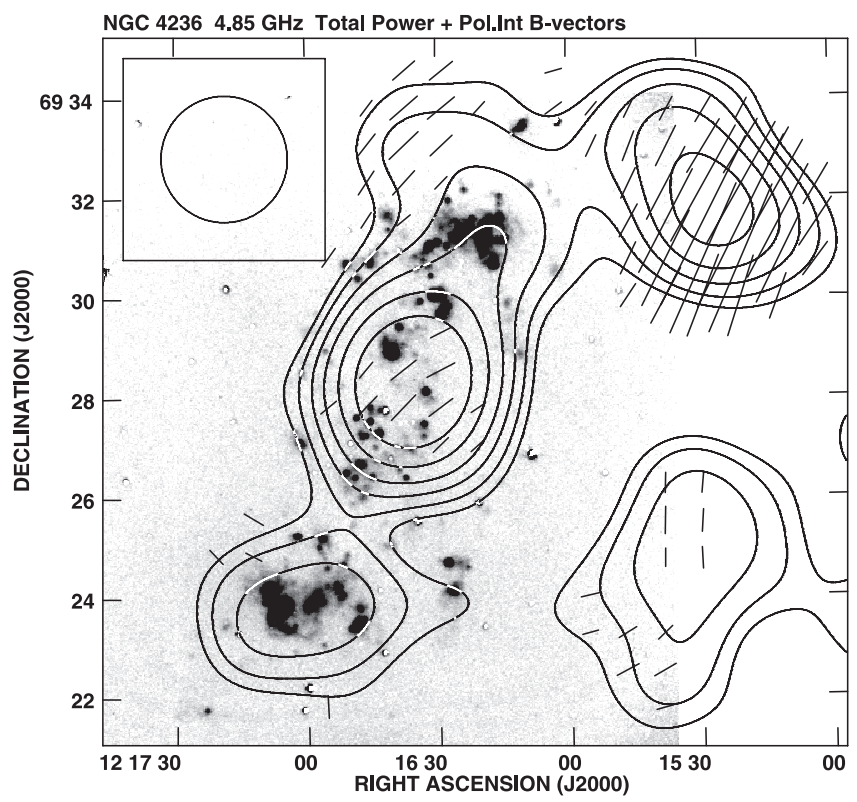

Fig. 2. Total power contours and $\boldsymbol{B}$-vectors of polarized intensity of NGC 4236 at $4.85 \mathrm{GHz}$ superimposed onto the $\mathrm{H} \alpha$ image. The contour levels are $(3,5,8,12,18,26) \times 0.3 \mathrm{mJy} / \mathrm{b}$.a. A vector of length of $1^{\prime}$ corresponds to a polarized intensity of $0.25 \mathrm{mJy} / \mathrm{b}$.a. The map resolution is $2.5 \mathrm{HPBW}$.

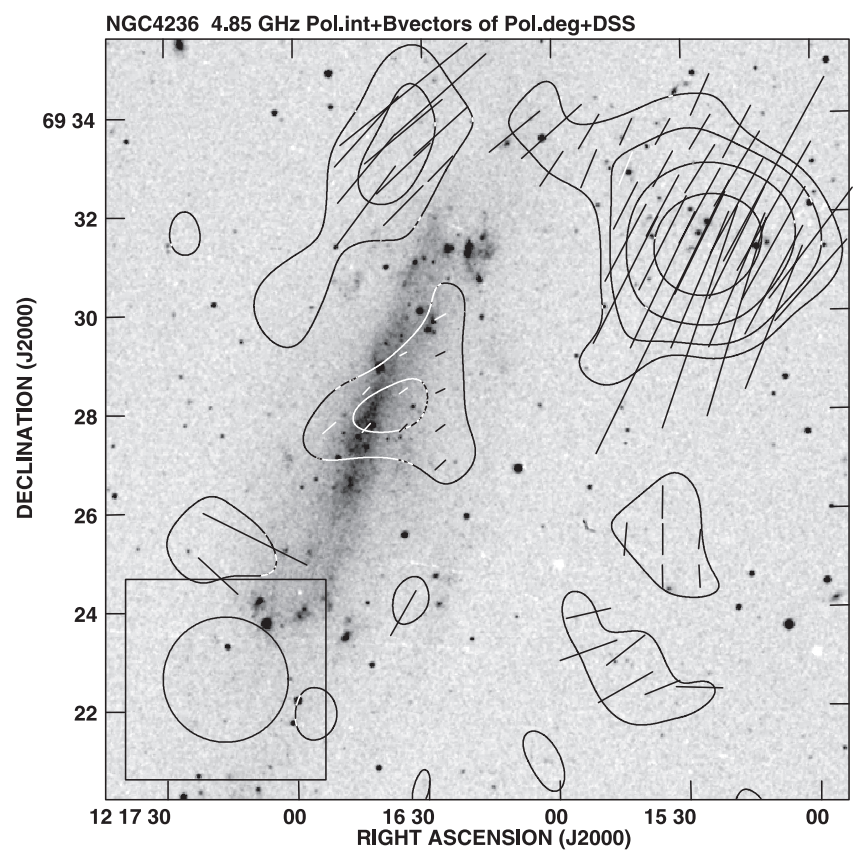

Fig. 3. Contours of polarized intensity and $\boldsymbol{B}$-vectors of polarization degree of NGC 4236 at $4.85 \mathrm{GHz}$ superimposed onto the blue image from DSS. The contour levels are $(2,4,8,16,32) \times 0.04 \mathrm{mJy} / \mathrm{b}$.a. A vector of length of $1^{\prime}$ corresponds to a polarization degree of $6.6 \%$. The map resolution is $2.5 \mathrm{HPBW}$.

towards the SE. In the NE half of the disk, the space between this ridge and the disk plane is filled with faint diffuse spurs.

The total power radio continuum of NGC 4656 at $4.85 \mathrm{GHz}$ (Fig. 5) shows two basic components: an elongated feature coincident with the NE part of the disk containing clusters of large $\mathrm{H}$ II regions and a bright unresolved source in the SW disk located within the optical limits of the galaxy. This source is found to be a very small object (less than few arcseconds in size) as

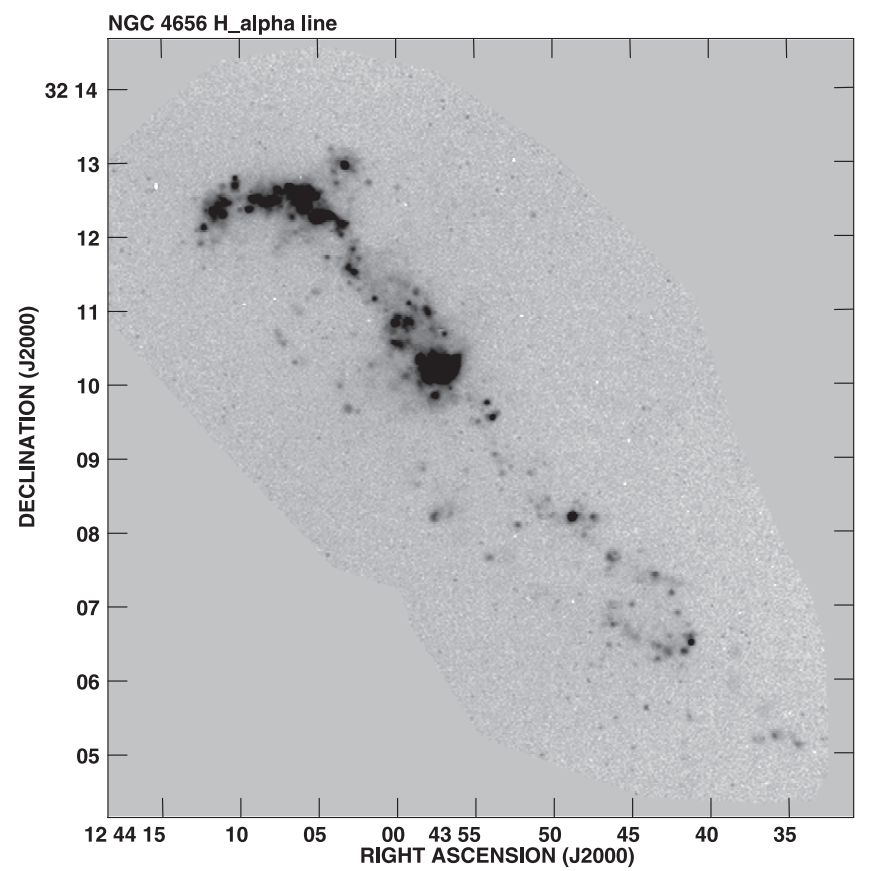

Fig. 4. The $\mathrm{H} \alpha$ image of NGC 4656 with the optical continuum subtracted.

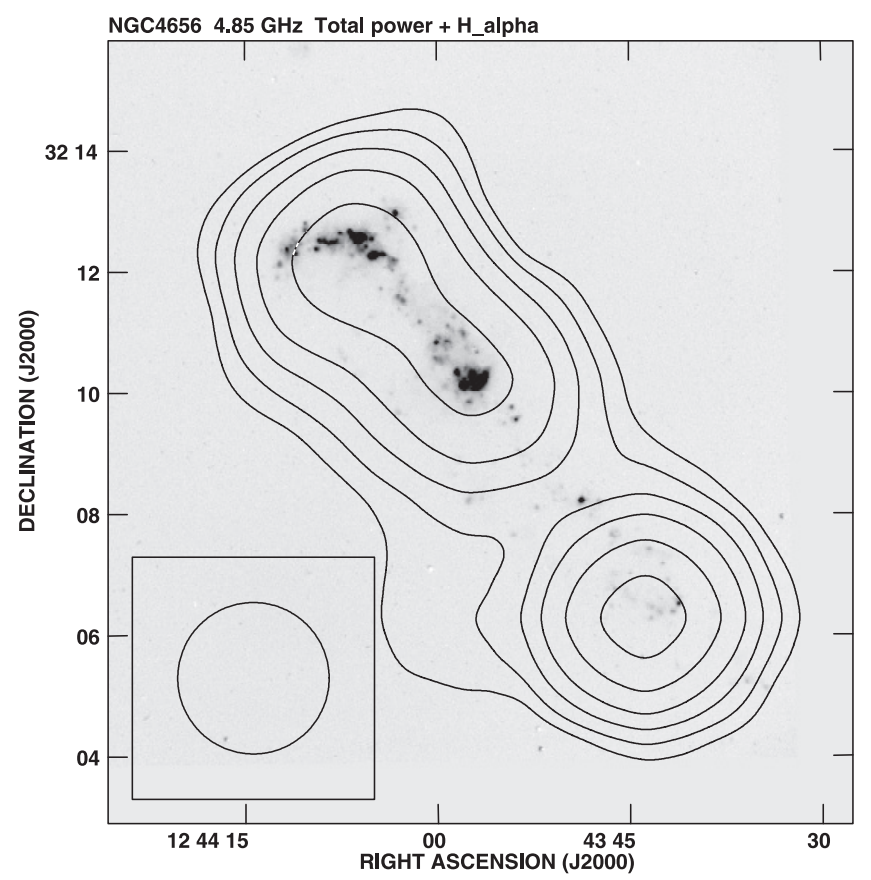

Fig. 5. Total power contours of NGC 4656 at $4.85 \mathrm{GHz}$ superimposed onto the $\mathrm{H} \alpha$ image. The contour levels are $(3,5,8,12,18,26) \times$ $0.65 \mathrm{mJy} / \mathrm{b}$.a. The map resolution is 2'.5 HPBW.

its flux density from NVSS (with 50" HPBW resolution) and from FIRST (resolution 10 times better) is almost the same. It is likely to be a very compact source with no optical counterpart at its well-measured (arcsec accuracy) position from FIRST. We assumed it to be a strong background source and we dropped it from further analyses.

The only obviously polarized feature in NGC 4656 is the peak of polarized intensity east of the northern disk boundary, south of the hook-like $\mathrm{H} \alpha$ and optical extension (Fig. 6). In this disk region we identified in the FIRST survey three background 


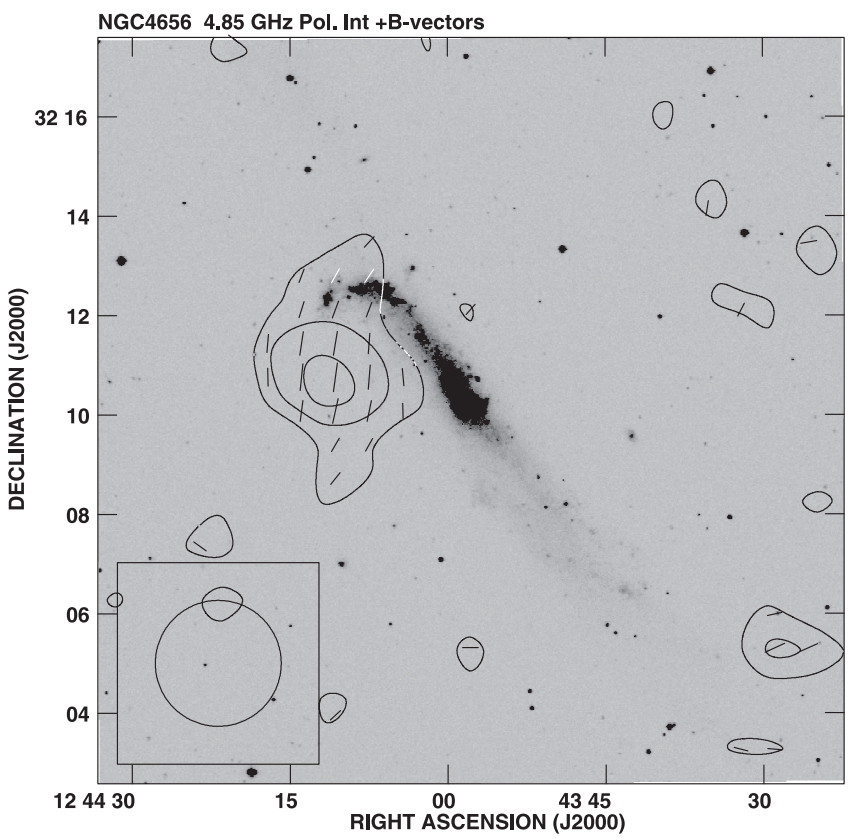

Fig. 6. Map of polarized intensity of NGC 4656 at $4.85 \mathrm{GHz}$ with $\boldsymbol{B}$-vectors of the same quantity overlaid upon the DSS red image. The contour levels are $(2,3,4,8,16) \times 0.08 \mathrm{mJy} / \mathrm{b}$.a. A vector of $1^{\prime}$ length corresponds to a polarized intensity of $0.59 \mathrm{mJy} / \mathrm{b}$.a. The map resolution is $2.5 \mathrm{HPBW}$.

sources, coinciding with the polarized peak. The whole polarization from this region was assumed to be due to background sources and dropped from further analyses, only the upper limit of the integrated polarized flux after its subtraction has been estimated. Integration of the total power map in the polygonal area encompassing all the visible radio emission yields an integrated flux density of NGC 4656 at $4.85 \mathrm{GHz}$ of $42 \pm 5 \mathrm{mJy}$. Integration of the map of polarized intensity over the same area as for the total power power emission yields only an upper limit for the polarized flux of $0.44 \mathrm{mJy}$ at 2-sigma confidence level. At $1.4 \mathrm{GHz}$ we obtain an integrated background-free flux density of $92 \pm 8 \mathrm{mJy}$.

\subsection{IC 2574}

The $\mathrm{H} \alpha$ emission in IC 2574 is almost entirely concentrated NE of the optical disk centre (Fig. 7). Its distribution corresponds very well to the optically bright star-forming regions. There is not much diffuse $\mathrm{H} \alpha$ emission in our map.

The area around the galaxy has a relatively low confusion level (unresolved background sources), thus we could attain an $\mathrm{rms}$ noise of $0.4 \mathrm{mJy} / \mathrm{b}$.a. The distribution of the radio continuum at $4.85 \mathrm{GHz}$ in IC 2574 is highly asymmetric with almost all radio emission concentrated in the vicinity of the NE star-forming clump (Fig. 8). Down to the mentioned rms level no emission has been found from the main galaxy's body.

In the case of IC 2574 there was no need to account our integrated flux densities for a contribution from background sources. Integration of the total power map in a polygonal area yields an integrated flux density of IC 2574 at $4.85 \mathrm{GHz}$ of $9.5 \pm 1.4 \mathrm{mJy}$. Integration of the map of polarized intensity in the same area results only in an upper limit of the polarized flux density of $0.46 \mathrm{mJy}$ at 2-sigma confidence level. At $1.4 \mathrm{GHz}$ we obtain an integrated, background-free flux density of $19 \pm 8 \mathrm{mJy}$.

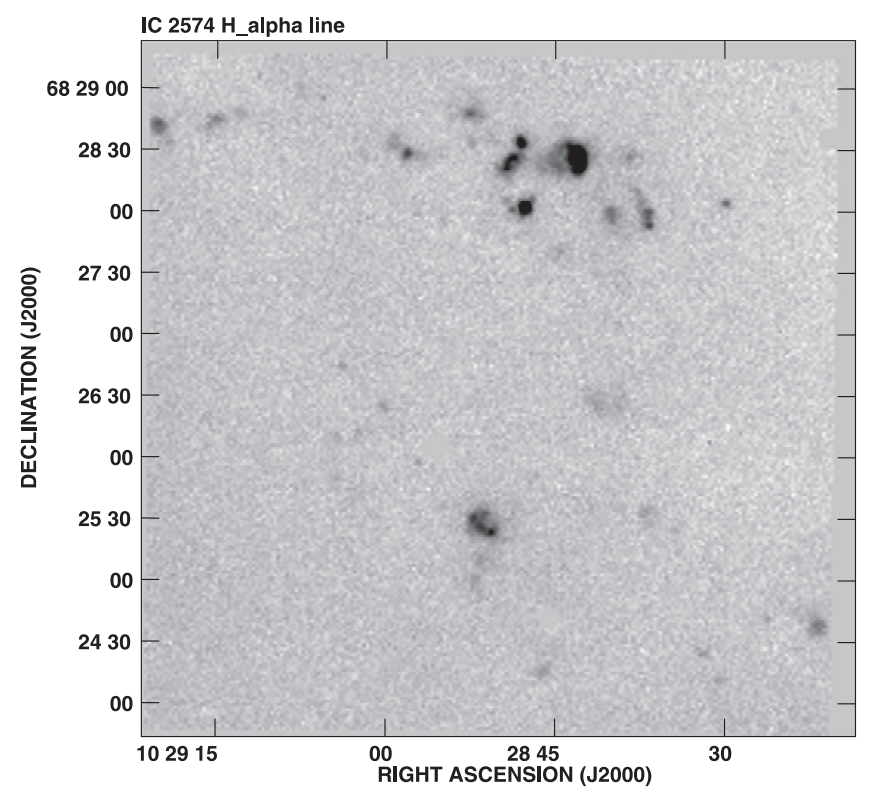

Fig. 7. $\mathrm{H} \alpha$ image of IC 2574 with the optical continuum subtracted. The map covers the most intense star formation regions in the northern part of the galaxy.

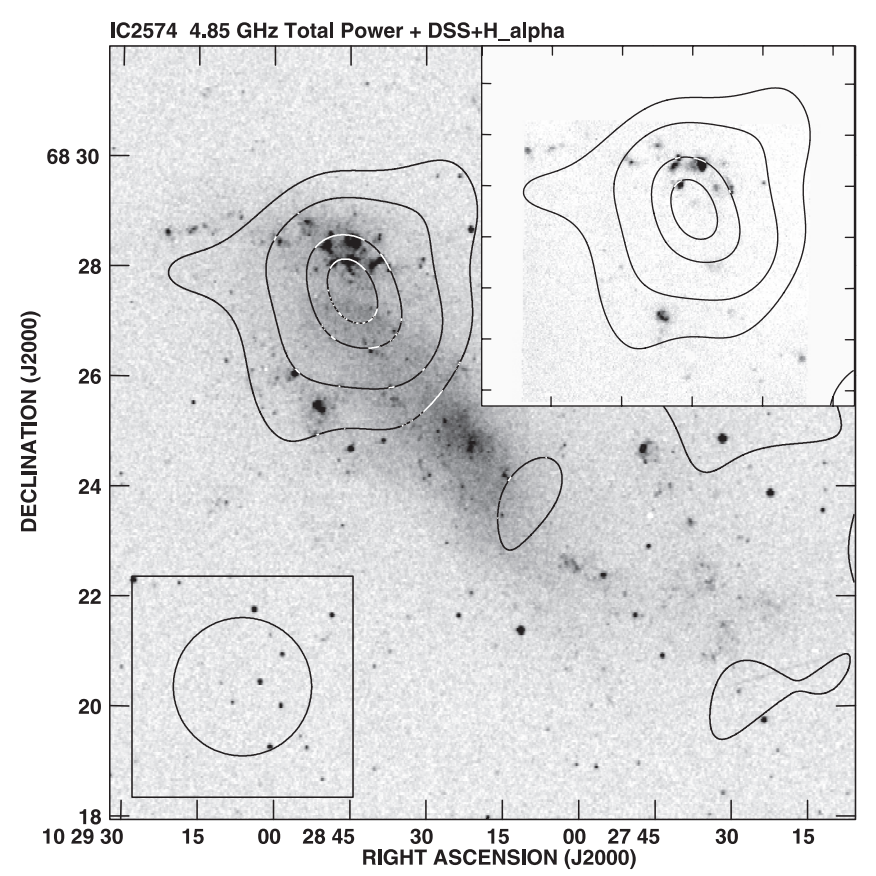

Fig. 8. Contour map of IC 2574 at $4.85 \mathrm{GHz}$ superimposed onto the blue DSS image. The contour levels are $(3.2,7,12,14) \times 0.35 \mathrm{mJy} / \mathrm{b}$.a. The map resolution is $2.5 \mathrm{HPBW}$. The inserted figure represents the same contour map overlaid on our $\mathrm{H} \alpha$ map covering the northern part of the galaxy.

\section{Discussion}

\subsection{The thermal emission}

We determined the thermal emission from the galaxies in our sample in two ways. First, we determined their radio spectral indices from the background-free integrated flux densities. Then we used a standard procedure of separating thermal and nonthermal emissions in the way similar to that described by Chyży et al. (2003). We assumed the nonthermal spectral index $\alpha_{\mathrm{nt}}$ subsequently equal to $0.8,0.9$ or 1.0 . The results: flux densities $S_{\text {tot }}$ 
at 4.85 and $1.4 \mathrm{GHz}$, observed spectral index $\alpha_{\mathrm{obs}}$, the assumed nonthermal one $\alpha_{\mathrm{nt}}$ as well as radio and optically derived thermal fluxes $S_{\text {th }}$ and thermal fractions $f_{\text {th }}$ are collected in Table 2.

We also determined the thermal flux and the thermal fraction using the calibrated $\mathrm{H} \alpha$ maps in the same way as in Chyży et al. (2003). To correct the $\mathrm{H} \alpha$ fluxes of our galaxies for absorption we used the total (galactic and internal) extinction corrections in $B$ and/or in $V$-colour as well as the reddening $E_{B-V}$ from the LEDA database. They are computed using the most recent data also including corrections for local anomalies. In converting them to the absorption in the red domain we compared several methods. First, we converted independently the $E_{B}$ and $E_{V}$ into $E_{R}$ using the appropriate "dust colours" from the NED database (assuming the colour of total absorption similar to that in our Galaxy). Alternatively we scaled $E_{B}$ and $E_{V}$ to $E_{R}$ using the extinction ratios from Schlegel et al. (1998). Additionally, we applied the statistical analyses of absorption by Cardelli et al. (1989) to $E_{B-V}$ taken from LEDA. The above variety of methods yielded thermal fractions in the range $0.45-0.63$ (NGC 4236), 0.5-0.9 (NGC 4656) and 0.62-0.78 for IC 2574. The mean values averaged over all methods, together with rms method-to-method scatter, are given in Table 2.

Niklas et al. (1997) gave the mean thermal fraction at $10.45 \mathrm{GHz} f_{\text {th }}=30 \% \pm 5 \%$ with $15 \%$ of the galaxies in their sample of 74 galaxies having $f_{\text {th }} \geq 45 \%$, independent of morphological type. They found a mean nonthermal spectral slope $\alpha_{\mathrm{nt}}=0.83$ for their sample of spiral galaxies. This implies a mean $f_{\text {th }} \simeq 24 \%$ at $4.85 \mathrm{GHz}$. Soida et al. $(2001,1996)$ simulated the radio spectra composed of assumed fractions of thermal and nonthermal emission with the slope of the latter adjusted to obtain the observed spectral index. They conclude that for the rapidly star-forming spirals NGC 3627 and NGC 4254 the model spectrum poorly fits observations if $f_{\text {th }}$ at $10.55 \mathrm{GHz}$ is higher than $40 \%$. This implies thermal fractions of these objects at $4.85 \mathrm{GHz}$ considerably lower than $25 \%$.

The uncertainties of both, the radio and optical method are quite large, because of a poor knowledge of nonthermal spectra and optical extinction. We note, however, that for two objects: NGC 4656 and IC 2574 the lowest thermal fractions obtained for various estimates of optical extinction are comparable to the radio-derived $f_{\text {th }}$ for the steepest nonthermal spectra. In NGC 4236 the ranges of the optically and radio-determined $f_{\text {th }}$ overlap, the optical ones also being somewhat higher. All this seems to favourize rather steep nonthermal spectra. Both the radio-derived thermal fractions assuming $\alpha_{\mathrm{nt}} \geq 0.9$ and lowest values from the $\mathrm{H} \alpha$ line are consistently higher by a factor of $\geq 1.9$ than the mean for radio-bright spirals studied by Niklas (1997). To make our estimates similar to those for normal spirals we would need to assume simultaneously that the nonthermal spectrum has a slope of $0.65-0.70$ (considerably flatter than for normal spirals) and that all our absorption estimates are too high by a factor of two. We consider such a coincidence as rather unlikely. It seems that the radio emission of our galaxies really shows a considerably increased thermal content compared to normal galaxies. The radio supernovae in NGC 4236 contribute some $8 \mathrm{mJy}$ to the total radio flux at $4.85 \mathrm{GHz}$ being themselves predominantly nonthermal point sources. Thus the thermal fraction of the diffuse emission from NGC 4236 may be even higher than given in Table 2 .

The highest thermal fraction in a spiral galaxy so far has been found in the edge-on spiral NGC 5907, i.e. $54 \%$ at $4.85 \mathrm{GHz}$ (Dumke et al. 2000). This galaxy has a rather low star formation rate and Dumke et al. (2000) proposed that the high thermal

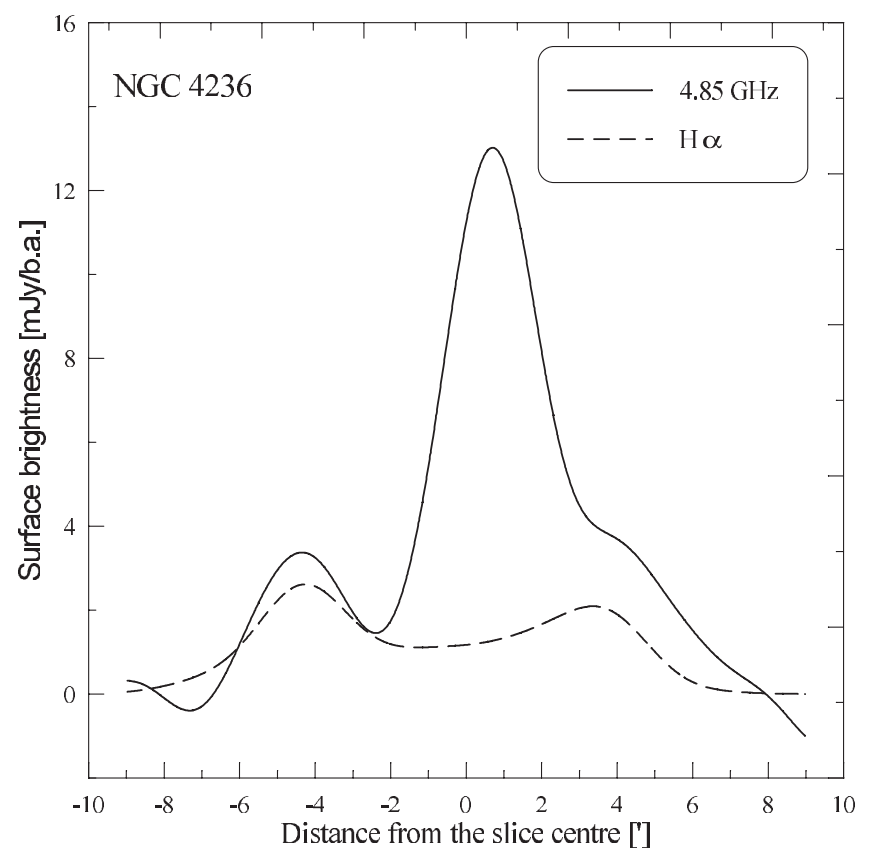

Fig. 9. Brightness profiles of the radio emission at $4.85 \mathrm{GHz}$ and of $\mathrm{H} \alpha$ emission along the disk plane of NGC 4236 . The slice centre is at $\mathrm{RA}_{2000}=12^{\mathrm{h}} 16^{\mathrm{m}} 39 \mathrm{~s} .7 \mathrm{Dec}_{2000}=+69^{\circ} 27^{\prime} 41^{\prime \prime}$ and the position angle is $334^{\circ} 6$. The $\mathrm{H} \alpha$ image is convolved to the resolution of the radio map of $2.5 \mathrm{HPBW}$.

fraction may not be due to an increased thermal emission but rather to a deficiency of nonthermal emission (see Sect. 4.4).

\subsection{Distribution of thermal and nonthermal emission}

Two of our galaxies (NGC 4236 and NGC 4656) possess clearly defined disks. For them we constructed profiles of total radio $(4.85 \mathrm{GHz})$ and thermal emission (derived from the $\mathrm{H} \alpha$ line) along the major axis. The $\mathrm{H} \alpha$ data were smoothed to the resolution of the radio maps (2.5). The profiles are centered on $\mathrm{RA}_{2000}=12^{\mathrm{h}} 16^{\mathrm{m}} 39.7 \mathrm{Dec}_{2000}=+69^{\circ} 27^{\prime} 41^{\prime \prime}$ and $\mathrm{RA}_{2000}=$ $12^{\mathrm{h}} 43^{\mathrm{m}} 58^{\mathrm{s}} .9 \mathrm{Dec}_{2000}=+32^{\circ} 10^{\prime} 32^{\prime \prime}$, respectively. A strong point source in the southern part of NGC 4656 has been subtracted. The profiles are shown in Figs. 9 and 10.

In NGC 4656 the distribution of total and thermal emission look similar (Fig. 10), with some minor asymmetry between the peaks. Along the whole profile the thermal fraction seems to undergo only small variations.

NGC 4236 looks different in this respect (Fig. 9). A strong, unresolved central radio peak has no counterpart in the $\mathrm{H} \alpha$ emission. Its amplitude can be well accounted for by the "radio supernovae" found there by Turner \& Ho (1994). In the remaining disk (e.g. at the distance of $5^{\prime}$ in Fig. 9) the thermal fraction may be very high, reaching some $70 \%$. This supports our conjecture from Sect. 4.1 that NGC 4236 may be dominated by thermal emission, having very weak global magnetic fields. We conclude that mechanisms producing the total magnetic fields in the presence of weak star formation are rather inefficient.

\subsection{Integrated properties}

Figure 11 shows the positions of our galaxies on a radio/FIR correlation between the surface brightness at $60 \mu \mathrm{m}$ and at $4.85 \mathrm{GHz}$ derived for a sample of normal spirals, measured at this frequency by Gioia et al. (1982, GGK). The fluxes at $60 \mu \mathrm{m}$ are 


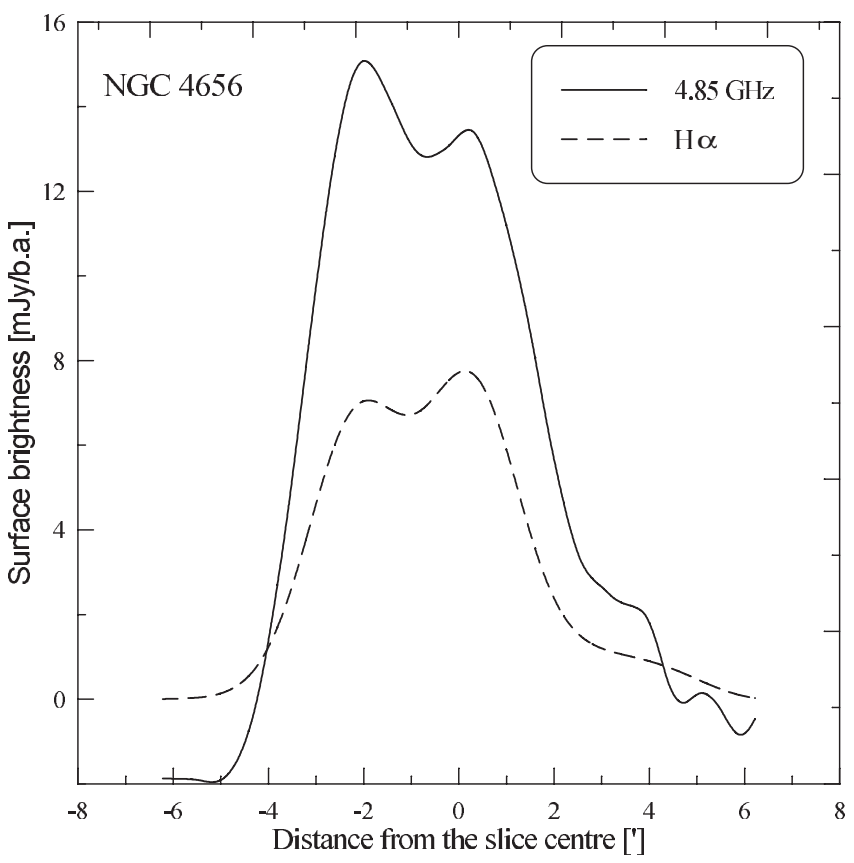

Fig. 10. Brightness profiles of the radio emission at $4.85 \mathrm{GHz}$ and of $\mathrm{H} \alpha$ emission along the disk plane of NGC 4656. The slice centre is at $\mathrm{RA}_{2000}=12^{\mathrm{h}} 43^{\mathrm{m}} 58^{\mathrm{s}} .9 \mathrm{Dec}_{2000}=+32^{\circ} 10^{\prime} 32^{\prime \prime}$ and the position angle is $221^{\circ}$. The $\mathrm{H} \alpha$ image is convolved to the resolution of the radio map of 2.5 HPBW.

taken from Helou \& Walker (1995) and if not present there from Moshir et al. (1990). The values of FIR and radio mean surface brightness have been obtained by dividing the corresponding integrated flux densities (in Jy) by the area within the extinction and inclination-corrected galaxy radius in the $B$ band at $25 \mathrm{mag} / \operatorname{arcsec}^{2}$ taken from the LEDA database. No correction for thermal emission has been made at this stage. As both the radio and FIR brightness are random variables we used the orthogonal fit to determine the slope of the best-fit line. In such case simple regressions of FIR vs. radio and radio vs. FIR give the minimum and maximum slope allowed by the data scatter, forming so-called "regression scissors". We used them as lower and upper limits to the slope of the best-fit line.

Our objects significantly extend the radio/FIR correlation at $4.85 \mathrm{GHz}$ towards the low surface brightness region. They follow well the correlation line with a slope of $0.96 \pm 0.06 \mathrm{de}-$ rived from the GGK sample alone. This means that the total (i.e. thermal and nonthermal) emission of our galaxies of low surface brightness follow the same correlation law as normal starforming, radio and FIR-bright spirals.

It is difficult to say whether the galaxies would lie below the correlation line for bright galaxies if only the nonthermal emission were considered. This needs a larger sample of galaxies spanning a broad range of star-forming activities, with radio spectra and $\mathrm{H} \alpha$ data analyzed in a homogeneous way. Such a systematic study is under consideration.

The mean degrees of nonthermal polarization (or their upper limits) of all galaxies of our sample are given in Table 3. We compared these rather low values with those of other highly-inclined spiral galaxies of similar angular size measured with the same angular resolution, hence influenced by beam depolarization in a similar way. These objects were observed at $4.85 \mathrm{GHz}$ with the $100-\mathrm{m}$ radio telescope by one of us (MK) in other works. Our comparison spirals: NGC 891, NGC 3628, NGC 4565 NGC5907 and NGC4631 have nonthermal

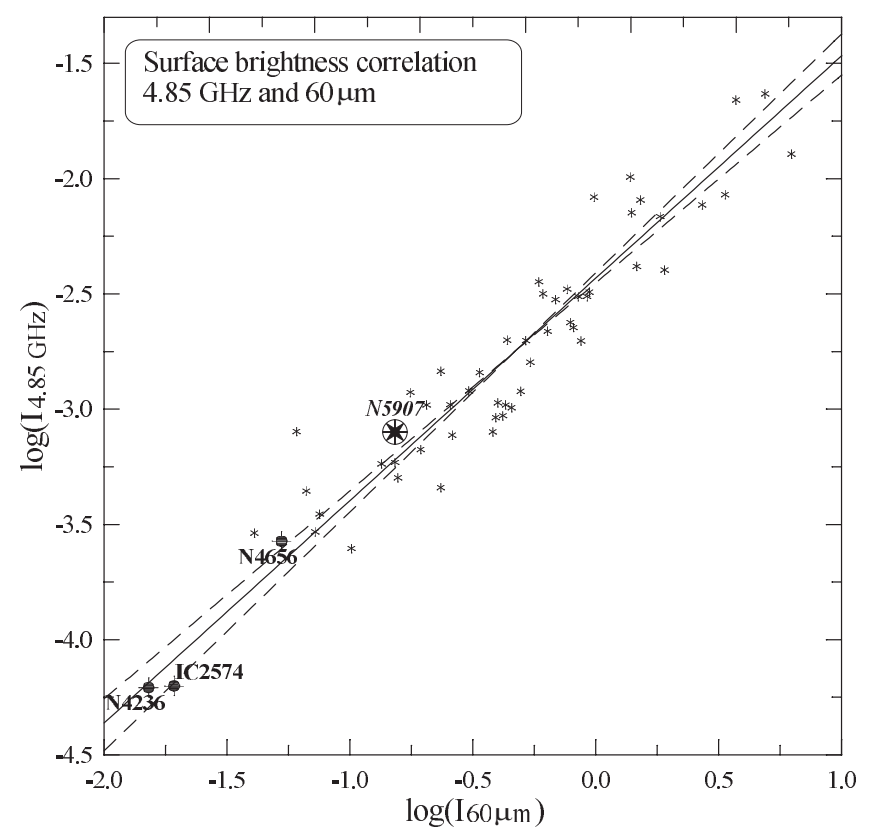

Fig. 11. Positions of the galaxies studied on the correlation diagram between the face-on corrected surface brightness at $60 \mu \mathrm{m}$ and at $4.85 \mathrm{GHz}$ (in Jy/ $\mathrm{arcmin}^{2}$ ). Small stars show the normally star-forming galaxies from the sample by Gioia et al. (1982), crossed dots - our objects. The solid line shows the orthogonal fit (slope 0.96) to objects from Gioia et al. (1982), dashed lines (slopes of 0.90 and 1.04) are the lower and upper limits to the best-fit slope (see text). A thick, encircled cross marks the position of NGC 5907, compared in the text to our galaxies.

polarization degrees of $3.2 \%, 5.3 \%, 13.7 \%, 3.2 \%$ and $4.9 \%$, respectively, assuming a mean thermal fraction for spirals of $20 \%$ at $4.85 \mathrm{GHz}$ (see Sect. 4.1). Adopting for NGC 4565 and NGC 5907, which have relatively low star formation, thermal fractions as high as $60 \%$ at $10.45 \mathrm{GHz}$ (Niklas et al. 1997) which yields $40 \%$ at $4.85 \mathrm{GHz}$, we obtain their nonthermal degrees of polarization $\simeq 17 \%$ and $4 \%$, respectively. The mean degree of polarization in NGC 4236 and the upper limit for NGC 4656 (Table 3) are below all these values. In NGC 4236 the polarization degree may be even lower if the bulk of polarization is due to the radio supernovae (see Sect. 3.1). We suggest that these two galaxies may have less ordered magnetic fields (hence regular fields weaker compared to total ones) than other more rapidly star-forming edge-on spirals. This means that also the mechanism producing regular magnetic fields may be less efficient in weakly star-forming galaxies. We note, however, that in case of IC 2574, a weak total power emission leads to a yet inconclusive upper limit for the polarization degree.

\subsection{Magnetic fields}

We computed the equipartition magnetic field strengths in our galaxies from the nonthermal surface brightnesses (according to Beck \& Krause 2005) in two ways. First, we used the mean value of the nonthermal surface brightness at $4.85 \mathrm{GHz}$ integrated over the whole extent of radio emission or (in case of IC 2574) over the optical extent. As a second approach, we took the mean nonthermal emission within the area delineated by $5 \%$ of the maximum signal or the area with emission above $2 \mathrm{rms}$ map level, taking the larger of these thresholds. We assumed a protonto-electron energy ratio of 100 . For each galaxy we used the nonthermal spectral index that yielded the best agreement between the radio and optically determined thermal fraction in Table 2. 
Table 3. Magnetic field strengths and degree of nonthermal polarization in the observed galaxies.

\begin{tabular}{lrrr}
\hline \hline Galaxy & $B_{\mathrm{t}}$ & $B_{\text {reg }}$ & Pol. degree \\
{$[\mu \mathrm{G}]$} & {$[\mu \mathrm{G}]$} & {$[\%]$} \\
\hline NGC 4236 total & $4.4 \pm 1.1$ & $0.6 \pm 0.2$ & $1.7 \pm 1.0$ \\
NGC 4236 5\% & $4.7 \pm 1.1$ & $0.7 \pm 0.2$ & $1.6 \pm 0.9$ \\
NGC 4656 total & $4.7 \pm 1.1$ & $<0.9 \pm 0.3$ & $<2.1^{a}$ \\
NGC 4656 2 rms & $4.9 \pm 1.2$ & $<1.0 \pm 0.3$ & $<2.0^{a}$ \\
IC 2574 total & $4.0 \pm 1.1$ & $<1.2 \pm 0.3$ & $<9.3^{a}$ \\
IC 2574 2 rms & $4.3 \pm 1.1$ & $<1.3 \pm 0.3$ & $<8.4^{a}$ \\
\hline
\end{tabular}

${ }^{a}$ Upper limit at the $2 \sigma$ level of integrated flux density uncertainty.

A synchrotron face-on disk thickness of $1 \mathrm{kpc}$ was assumed. We determined the average brightness of the radio emitting region in IC 2574 by taking it roughly spherical with a diameter of $4.5 \mathrm{kpc}$. The resulting total and regular magnetic field strengths as well as the mean degree of nonthermal polarization (corrected for polarized background sources) are shown in Table 3. Their errors include the $50 \%$ uncertainties of the above parameter values.

Our galaxies have relatively low total mean magnetic field strengths (in the range 4.0 to $4.9 \mu \mathrm{G}$ ) when compared to normal spirals (for which the mean value depending on the galaxy sample is $8-10 \mu \mathrm{G}$, Beck et al. 1996). In NGC 4236 the mean total "diffuse" magnetic field may be even weaker if the "radio supernovae" discussed by Turner \& Ho (1994) and in Sect. 4.1 are removed.

The galaxies of our sample belong to objects with the weakest total fields so far measured in galaxies. Other spirals with weak total and regular magnetic fields are e.g. NGC 5907 (Dumke et al. 2000) and the Sombrero galaxy M 104 (Krause et al. 2006). Although the former is a late type spiral and the latter an early-type spiral, both have low star formation rates. Furthermore, like the galaxies of our sample, NGC 5907 has a high thermal fraction which may be due to a deficiency of synchrotron emission, which in turn indicates a low magnetic field strength. A similar deficiency of synchrotron emission seems to be present in galaxies studied in this work.

According to Dumke et al. (2000) a higher star formation activity may lead to a much more significant increase of the number density of relativistic electrons and a more effective amplification of the magnetic fields, and therefore to a higher fraction of nonthermal (synchrotron) radiation than for galaxies with a low star-formation rate (SFR). A more efficient magnetic field generation is predicted e.g. by the model of the dynamo process driven by Parker-type magnetic instabilities boosted by star-formation (Hanasz et al. 2004). The pressure of cosmic rays produced by supernovae accelerates the instabilities which in turn leads to a rapid amplification of both total and regular magnetic fields (the latter by fast reconnection). This scenario would be consistent with the situation in the galaxies in our sample: they all have a high thermal fraction, weak total emission, and low star formation activity. Indeed, Niklas et al. (1995) found that the lowest thermal fractions occur in galaxies with the highest SFR.

Niklas \& Beck (1997) explained the radio-FIR correlation by a simple model where the energies of turbulent gas motions, magnetic fields and cosmic rays are in equipartition. As a result, the field strength $B_{\mathrm{t}}$ increases as $B_{\mathrm{t}} \propto S F R^{1 /(2 N)}$ where $N$ is the exponent of the Schmidt law ( $S F R \propto \rho^{N}$, Schmidt 1959), and the nonthermal surface brightness $I_{\text {nth }}$ increases as $I_{\text {nth }} \propto$ $S F R^{\left(3+\alpha_{\mathrm{nt}} / /(2 N)\right.}$. For $N \simeq 1.4$ and $\alpha_{\mathrm{nt}}=0.9$ we get $I_{\mathrm{nth}} \propto S F R^{\approx 1.4}$. As the thermal radio surface brightness is expected to increase linearly with $S F R$, the ratio of nonthermal to thermal emission increases with $S F R \approx 0.4$. This simple model can basically explain our result that the nonthermal radio emission and the ratio of nonthermal to thermal emission are nonlinear functions of the star-formation rate. Below some threshold in star-formation activity, the dynamo may not work efficiently anymore, and the nonthermal emission may drop further. Below some low value of $S F R$, the radio emission may be almost completely thermal, but the radio-FIR correlation is still valid. A detailed study of these correlations is in preparation.

\section{Summary and conclusions}

We observed the three angularly large, late-type, slowly starforming nearby galaxies NGC 4236, NGC 4656 and IC 2574 with the Effelsberg radio telescope at $4.85 \mathrm{GHz}$ in order to check whether they still host widespread magnetic fields. Observations at $1.4 \mathrm{GHz}$ were also made to determine the radio spectra. Our results were carefully accounted for possible background sources. We also observed these galaxies in the $\mathrm{H} \alpha$ line to study the distribution of recent star formation and to estimate thermal fractions independent of the radio spectrum. The most important results are as follows:

- The galaxies possess a very clumpy distribution of the $\mathrm{H} \alpha$-emitting gas. The $\mathrm{H}$ II regions are very non-uniformly distributed being concentrated in selected parts of the galaxies in a very asymmetric way. NGC 4236 shows even some extraplanar $\mathrm{H} \alpha$ emission.

- A reasonable agreement between mean thermal fractions determined from the radio spectrum and from the extinctioncorrected $\mathrm{H} \alpha$ line was attained. The galaxies show thermal fractions at $4.85 \mathrm{GHz}$ considerably higher than most normal spirals, reaching $0.5-0.7$ (depending on the assumptions). This effect is most pronounced for the radio-weakest objects.

- The galaxies clearly show diffuse nonthermal emission indicative of widespread magnetic fields. Their mean total surface radio brightness is however smaller than in most normally star-forming intermediate-type spirals.

- The fraction of nonthermal emission in our objects is smaller than for normally star-forming spirals which suggests that magnetic fields are even weaker than one could expect from their low radio brightness. Their total magnetic fields have mean strengths of $4.0-4.9 \mu \mathrm{G}$. This result can be explained in terms of the equipartition model between turbulent gas motions, magnetic fields and cosmic rays, in which the nonthermal emission increases faster than linearly with the $S F R\left(\propto S F R^{\approx 1.4}\right)$ and that the ratio of nonthermal to thermal emission increases with $S F R^{\approx 0.4}$.

- The galaxies still fall on the radio-FIR correlation for the total surface brightness, following the same slope of $0.96 \pm 0.06$ as radio-bright spirals. Our sample extends the correlation to the lowest values observed so far.

- Weak polarization at $4.85 \mathrm{GHz}$ attributable to the galaxy has been detected only in NGC 4236. Even in this case it can be due to few young supernova remnants. Two galaxies (NGC 4236 and NGC 4656) have a nonthermal polarization degree below values measured for other edge-on spirals at $4.85 \mathrm{GHz}$ with a similar resolution. The degree of magnetic field regularity of these two weakly star-forming spirals is lower than that of normally star-forming and rapidly rotating edge-on spirals. The third object (IC 2574) is too weak for any definite constraints.

In this work we demonstrated that the slowly rotating and weakly star-forming spirals observed by us may possess 
widespread magnetic fields. The field generation mechanism, especially that of the regular magnetic field, seems to be less efficient in these objects than in rapidly star-forming spirals. This gives arguments for magnetic field generation theories involving unstable processes (like Parker instabilities) energized by star-forming processes. To what extent these results apply to slowly star-forming galaxies in general will be a subject of future projects dealing with a larger sample of objects.

Acknowledgements. The authors, (K.Ch., M.S. and M.U.) are indebted to Professor Richard Wielebinski from the Max-Planck-Institut für Radioastronomie (MPIfR) in Bonn for the invitations to stay at this institute where substantial parts of this work were done. A large part of the work has been done in the framework of the exchange program between the Jagiellonian University and Ruhr-Universität Bochum. We are grateful to Dr. Elly Berkhuijsen for valuable comments and careful reading of the manuscript. We also would like to thank an anonymous referee for his comments. We acknowledge the usage of the HyperLeda database (http://leda.univ-lyon1.fr), the NRAO VLA Sky Survey (NVSS) and Faint Images of the Radio Sky at Twenty-cm (FIRST) survey. This work was supported by a grant No. PB0249/P03/2001/21 from the Polish Research Committee (KBN).

\section{References}

Baars, J. W. M., Genzel, R., Pauliny-Toth, I. I. K., \& Witzel, A. 1977, A\&A, 61, 99

Beck, R., \& Krause, M. 2005, Astr. Nachr., 326, 414

Beck, R., Brandenburg, A., Moss, D., Shukurov, A., \& Sokoloff, D. 1996, ARA\&A, 34, 155

Blackman, E. G., 1998, ApJ, 496, L17

Bomans, D. J., Chu, Y. H., \& Hopp, U. 1997, AJ, 113, 1678

Cardelli, J. A., Clayton, G. C., \& Mathis, J. S. 1989, ApJ, 345, 245

Chyży, K. T., Beck, R., Kohle, S., Klein, U., \& Urbanik, M. 2000, A\&A, 355, 128
Chyży, K. T., Knapik, J., Bomans, D. J., et al. 2003, A\&A, 405, 513

Condon, J. J. 1987, ApJS, 65, 485

Dumke, M., Krause, M., \& Wielebinski, R. 2000, A\&A, 355, 512

Emerson, D. T., \& Gräve R. 1988, A\&A, 190, 353

Emerson, D. T., Klein, U., \& Haslam, C. G. T. 1979, A\&A, 76, 92

Gioia, I. M., Gregorini, L., \& Klein, U. 1982, A\&A, 116, 164

Hanasz, M., Kowal, G., Otmianowska-Mazur, K., \& Lesch, H. 2004, ApJ, 605, L33

Haslam, C. G. T. 1974, A\&AS, 15, 333

Helou, G., \& Walker, D. W. 1995, IRAS Small Scale Structure Catalog

Ho, L. C., Filippenko, A. V., \& Sargent, W. L. 1998, ApJS, 98, 477

Honma, M., \& Sofue, Y. 1997, PASJ, 49, 539

Karachentsev, I. D., Dolphin, A. E., Geisler, D., et al. 2002, A\&A, 383, 125

Klein, U., Weiland, H., \& Brinks, E. 1991, A\&A, 246, 323

Kowal, G., Hanasz, M., \& Otmianowska-Mazur K. 2003, A\&A, 404, 533

Krause, M., Wielebinski, R., \& Dumke, M. 2006, A\&A, 448, 133

Martimbeau, N., Carigman, C., \& Roy, J.-R. 1994, AJ, 107, 543

Morsi, H. W., \& Reich, W. 1986, A\&A, 163, 313

Moshir, M., Copan, G., Conrow, et al. 1990, IRAS Faint Source Catalogue, version 2.0, Vizier Online Data Catalog, 2156, 0

Moss, D., Shukurov, A., \& Sokoloff, D. 1999, A\&A, 343, 120

Niklas, S., 1995, Ph.D. Thesis, University of Bonn

Niklas, S., \& Beck, R. 1997, A\&A, 320, 54

Niklas, S., Klein, U., \& Wielebinski, R. 1997, A\&A, 322, 19

Rand, R. J. 1994, A\&A, 285, 833

Schekochihin, A. A., Cowley, S. C., Taylor, S. F., Maron, J. L., \& McWilliams, J. C. 2004, ApJ, 612, 276

Schmidt, M. 1959, ApJ, 129, 24

Schmidt, A., Wongsowijoto, A., Lochner, O., et al. 1993, MPIfR Technical Report No. 73, MPIfR, Bonn

Schlegel, D. J., Finkbeiner, D. P., \& Davis, M. 1998, ApJ, 500, 525

Soida, M., Urbanik, M., \& Beck, R. 1996, A\&A, 312, 409

Soida, M., Urbanik, M., Beck, R., Wielebinski, R., \& Balkowski, C. 2001, A\&A, $378,40 \mathrm{~S}$

Tabara, H., \& Inoue, M. 1980, A\&AS, 39, 379

Turner, J. L., \& Ho, P. T. P. 1994, ApJ, 421,122 